\title{
Moving Next-Generation Sequencing into the Clinic
}

\author{
Omshree Shetty ${ }^{1}$ Mamta Gurav ${ }^{1}$ Prachi Bapat ${ }^{1}$ \\ Sridhar Epari ${ }^{2}$ Sangeeta Desai \\ ${ }^{1}$ Division of Molecular Pathology, Department of Pathology, Tata \\ Memorial Centre, Homi Bhabha National Institute, Mumbai, \\ Maharashtra, India \\ ${ }^{2}$ Department of Pathology, Tata Memorial Centre, Homi Bhabha \\ National Institute, Mumbai, Maharashtra, India
}

\author{
Nupur Karnik² Gauri Wagh ${ }^{2}$ Trupti Pai ${ }^{2}$
}

Address for correspondence Omshree Shetty, PhD, Molecular Pathology Laboratory, Annexe Building, Tata Memorial Centre, Homi Bhabha National Institute, Mumbai 400012, Maharashtra, India (e-mail: omshreens@gmail.com, shettyoa@tmc.gov.in).

\begin{abstract}
Keywords

- molecular diagnostics

- NGS

- oncology
\end{abstract}

\section{Introduction}

The unraveling of clinically important biomarkers as a result of profound characterization of oncogenome has necessitated the screening of multiple genes in tumors as the benchmark of care. The revolutionary next-generation sequencing (NGS) technologies due to their massively parallel sequencing abilities enable the simultaneous screening of multiple genes in multiple samples. NGS has the ability to provide simultaneous screening of a wide range of genomic aberrations such as single-nucleotide variants (SNVs), multiple-nucleotide variants (MNVs), frameshift alterations, large insertion and deletions (INDEL), and copy number variation (CNVs) of the genes. In NGS, on an average, the targeted areas of importance are repeatedly sequenced with high throughput, providing high sensitivity and confidence for mutation detection. These advantages make it a very promising tool for implementing in routine clinical diagnostics.
DOI https://doi.org/ $10.1055 / \mathrm{s}-0041-1732854$ ISSN 0971-5851
(C) 2021. Indian Society of Medical and Paediatric Oncology. This is an open access article published by Thieme under the terms of the Creative Commons Attribution-NonDerivative-NonCommercial-License, permitting copying and reproduction so long as the original work is given appropriate credit. Contents may not be used for commercial purposes, or adapted, remixed, transformed or built upon. (https://creativecommons.org/licenses/by-nc-nd/4.0/). Thieme Medical and Scientific Publishers Private Ltd. A-12, Second Floor, Sector -2, NOIDA -201301, India 
However, the challenges are myriad and range from learning basic technology, overall fiscal implications, standardization, validation, data analysis and interpretation, and using the test result in the clinic. ${ }^{1}$

NGS assays can be developed to target the genome at various levels (whole genome, whole exome, and targeted panels) and prove to be an essential component toward realizing effective stratified oncology. There is a considerable variety across targeted NGS in terms of choice of panels, method of detection, and amount of input material. All these variabilities offer complexities in adapting this technology into routine practice.

The current article describes the processes of setting up, validation, and data analysis of NGS, including the stepwise guidance for setting up the NGS testing assays. The outline of the article would be as follows:

1. Types of NGS assays:

- Whole genome sequencing

- Whole exome sequencing

- Transcriptome sequencing

- Targeted sequencing

2. Sequencing platforms/sequencing chemistry

3. Basic NGS workflow:

- Preanalytical factors

- Target enrichment techniques

- Analytical factors

- Nucleic acid quality and quantification

- Quality and quantification of library preparation

- Bioinformatics pipeline

- Variant interpretations

- Interpretation and reporting recommendations

- Validation of NGS runs

4. Important points to consider

\section{Types of Next-Generation Sequencing Assays}

- Whole genome sequencing (WGS): It is the largest parallel sequencing of the entire genome of an organism which yields tremendous data output but currently relevant only in research settings and not feasible for clinical diagnostics.

- Whole exome sequencing (WES): This is a technique for sequencing all of the protein-coding transcripts of genes (known as exome). WES is an effective method for detection of possible pathogenic alterations. However, it tends to miss DNA variations outside the exons. Similar to WGS, it is mainly used in research settings and is useful in determining new genetic variations associated with diseases. Analytical specificity may also be compromised with shallow coverage and hence is not a preferred choice when it comes to clinical utility in diagnostic settings. ${ }^{2}$

- Whole transcriptome sequencing (RNA sequencing): This technique examines the sequences of RNA and analyses the transcriptome of gene expression patterns encoded within the RNA. It is a promising alternative to microarray and has several advantages, for example, greater dynamic range, increased analytical sensitivity toward underexpressed genes, alternative splicing detection, and gene fusion discovery. However, its clinical utility is not feasible. ${ }^{3}$

- Targeted disease specific panels: In routine clinical practice, the NGS test for genotyping requires careful design and should be technically reproducible and reliable. The inclusion of candidate genes, targeted region of interest, and actionable variants need to be integrated in the design of NGS test. ${ }^{4}$ Targeted sequencing is a popular method to detect known and novel variants in selected sets of genes or mutational hotspots. It is a rapid and a cost-effective way with well-established studies on sensitivity, specificity, precision, and reproducibility of such panel-based testing in clinical settings. ${ }^{5}$ The U.S. Center for Disease Control and Prevention (CDC), American College of Medical Genetics and Genomics (ACMG), Association for Molecular Pathology (AMP), and College of American Pathologists (CAP) have developed guidelines for panel-based somatic mutation testing. Focusing on a limited set of genes allows for deeper coverage of precise information and, more importantly, is very economical, making it user friendly, especially for diagnostic settings. ${ }^{2,6,7,8,9}$

DNA/RNA combination panels: These target panels range from $<10$ genes to approximately 100 genes comprising of all the predominant hotspot alterations specified for most commonly occurring solid tumors. For CNV, SNV, frameshift mutations, INDELs, and Micro satellite Instability (MSI) DNA-based NGS panels are used. Similarly, for detecting rearrangements, fusion RNA panel is used. These panels can be customized or made available commercially. Use of combination panel comprising of both DNA and RNA together is the most preferred panel, as it gives comprehensive information, as well as saves time in performing the assay and economical as well. On the same flow cell, both DNA and RNA libraries can be loaded simultaneously.

Consensus recommendation on NGS testing indicates that "actionable gene" variations affects clinical decisions, thereby guiding the clinicians in offering correct diagnosis and planning treatment protocol based on these findings. Numerous studies are being pursued on mechanism of tumorigenesis, making it of utmost important to address the gene list regularly based on the continual progress on available scientific data as depicted in - Table $1 .^{10}$

\section{Sequencing Platforms}

Currently available commercial platforms are based on the principle to perform massively parallel chemical reactions in such a way that allows the individual product to be analyzed. These different chemistries, including sequencing by synthesis or by ligation sequencing with reversible terminators, bead capture, and ion sensing, are the various library preparation methods. Each platform has unique need-based parameters to the laboratory and test requirements including instrument size, cost, run time, panel size, read length, 
Table 1 The most commonly used candidate genes in solid tumor targeted NGS panels

\begin{tabular}{|l|l|l|l|}
\hline Driver gene variants & Type of alterations & Disease & Evidence \\
\hline $\begin{array}{l}\text { EGFR, KRAS, BRAF, MET } \\
\text { ALK, ROS, RET }\end{array}$ & $\begin{array}{l}\text { SNV } \\
\text { Fusion }\end{array}$ & $\begin{array}{l}\text { Lung cancer } \\
\text { Rearrangements }\end{array}$ & NCCN, DLCG, NHFPCDT \\
\hline MET Exon 14 skipping & Amplification, SNV & Breast cancer & High level, wide acceptance \\
\hline HER2, BRCA1, BRCA2, ESR1 & $\begin{array}{l}\text { Amplification, SNV, CNV frameshift } \\
\text { alterations }\end{array}$ & Gastric cancer & Low level, wide acceptance \\
\hline $\begin{array}{l}\text { HER2, } \\
\text { SMAD4, STK11, APC,KIT, PDGFRA }\end{array}$ & SNV, CNV & $\begin{array}{l}\text { Hepatocellular } \\
\text { carcinoma }\end{array}$ & Low level wide acceptance \\
\hline $\begin{array}{l}\text { TP53, IDH1, IDH2, FGF, KRAS } \\
\text { KRAS, NRAS, BRAF and MSI } \\
\text { related genes like MLH1, MSH2, }\end{array}$ & SNV, amplifications & Colorectal cancer & NCCN \\
\hline
\end{tabular}

Abbreviations: NCCN, National Comprehensive Cancer Network DLCG, Danish Lung Cancer Group; NHFPCDT, National Health and Family Planning Commission of the People's Republic of China CNV, copy number variation; NGS, next-generation sequencing; SNV, single-nucleotide variants.

Table 2 Different types of sequencing platforms and the method of sequencing

\begin{tabular}{|c|c|c|c|}
\hline Platform & Method of Sequencing & Pros & Cons \\
\hline Illumina & $\begin{array}{l}\text { Sequencing by synthesis-cyclic } \\
\text { reversible termination technology } \\
\text { Paired end sequencing }\end{array}$ & $\begin{array}{l}\text { High throughput, low cost per } \\
\text { Gb data. High accuracy }\end{array}$ & $\begin{array}{l}\text { Short read length, high instru- } \\
\text { ment cost }\end{array}$ \\
\hline Ion Torrent & $\begin{array}{l}\text { lon semiconductor sequencing } \\
\text { technology }\end{array}$ & $\begin{array}{l}\text { Low instrumental and oper- } \\
\text { ational cost. Short execution } \\
\text { time. Simple machine }\end{array}$ & $\begin{array}{l}\text { High error rate. Intermediate cost } \\
\text { per Gb data. More hands-on time }\end{array}$ \\
\hline Pacific Bioscience & $\begin{array}{l}\text { Single-molecule real-time long-read } \\
\text { sequencing }\end{array}$ & $\begin{array}{l}\text { Longest reading length availa- } \\
\text { ble. Short instrument execution } \\
\text { time }\end{array}$ & $\begin{array}{l}\text { High error rate. High cost per } \\
\text { Gb data. Many methods are still } \\
\text { under development }\end{array}$ \\
\hline Oxford Nanopore & $\begin{array}{l}\text { Single-molecule real-time long read } \\
\text { sequencing }\end{array}$ & $\begin{array}{l}\text { Small, portable and low cost } \\
\text { instrument }\end{array}$ & $\begin{array}{l}\text { High error rate. Biased errors. } \\
\text { High cost per reading }\end{array}$ \\
\hline Complete Genomics BGI & $\begin{array}{l}\text { Sequencing by ligation } \\
\text { DNA nanoball sequencing mate pair } \\
\text { library }\end{array}$ & $\begin{array}{l}\text { Low cost per run, short execu- } \\
\text { tion time, large amount of data, } \\
\text { error rates low }\end{array}$ & Short read length \\
\hline Gene Reader (Qiagen) & $\begin{array}{l}\text { Sequencing by synthesis, single } \\
\text { nucleotide addition }\end{array}$ & $\begin{array}{l}\text { High throughput, reduced } \\
\text { manual intervention, complete } \\
\text { solution from extraction to data } \\
\text { analysis }\end{array}$ & Closed system, high cost \\
\hline
\end{tabular}

and actual cost per test. Depending on the clinical utility, availability of funds and infrastructure, the laboratory can decide on whichever platform is suitable for use. The detailed description of the pros and cons of different sequencing platforms has been mentioned in - Table 2 .

\section{Next-Generation Sequencing Workflow Quality Parameters}

NGS is a multistep assay that starts with the identification of the optimum sample and tumor enrichment followed by target enrichment and library preparation, followed by massive parallel sequencing and data output and subsequent analysis. The NGS workflow has been outlined in -Fig. 1 .

\section{Preanalytical Factors}

The sample type frequently used for analysis of solid tumors is formalin-fixed paraffin-embedded (FFPE) tissue. The hematoxylin and eosin (H\&E)-stained slides of the representative tumor sections are reviewed to quantify approximate tumor content. In case of accompanying necrosis or more of normal tissue content, macrodissection can be done for tumor enrichment before nucleic acid extraction. On completion of nucleic acid extraction, a majority of NGS assay setup requires a quality control step wherein ultraviolet (UV) spectrophotometry can be used for quality assessment and fluorometric methods for quantification. The step following this is the preparation of the library which is the most important step. Sequencing libraries are usually prepared by fragmentation of DNA and adding unique adapters at both the sides of the fragments.

Analytical factors: Optimum nucleic acid extraction is the main key in obtaining the successful NGS results. Guidelines which enumerate the validation protocol, as well as a list of different specimens, and extraction methods which approved and validated for NGS testing are available. ${ }^{8,11}$ For a successful NGS setup, 50 to $60 \mathrm{ng}$ of good quality DNA/RNA is required as a starting material. ${ }^{6}$ This nucleic acid is used for target enrichment and subsequently for library preparation. Different approaches used for target enrichment constitute an important analytical parameter. 


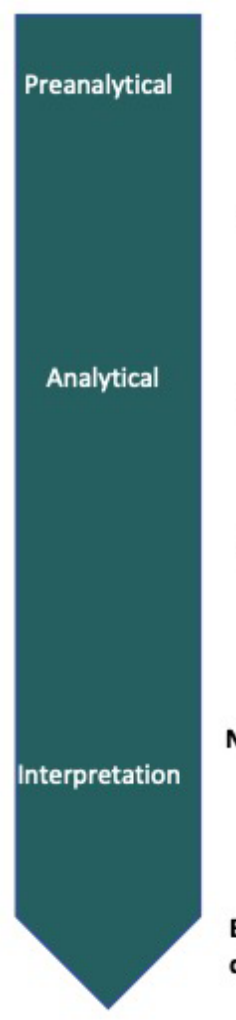

Sample Source

Molecular Targets

Normalization

Biomarker determination
Output

\section{Analyses}

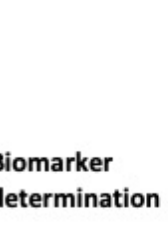

Tumor Sample
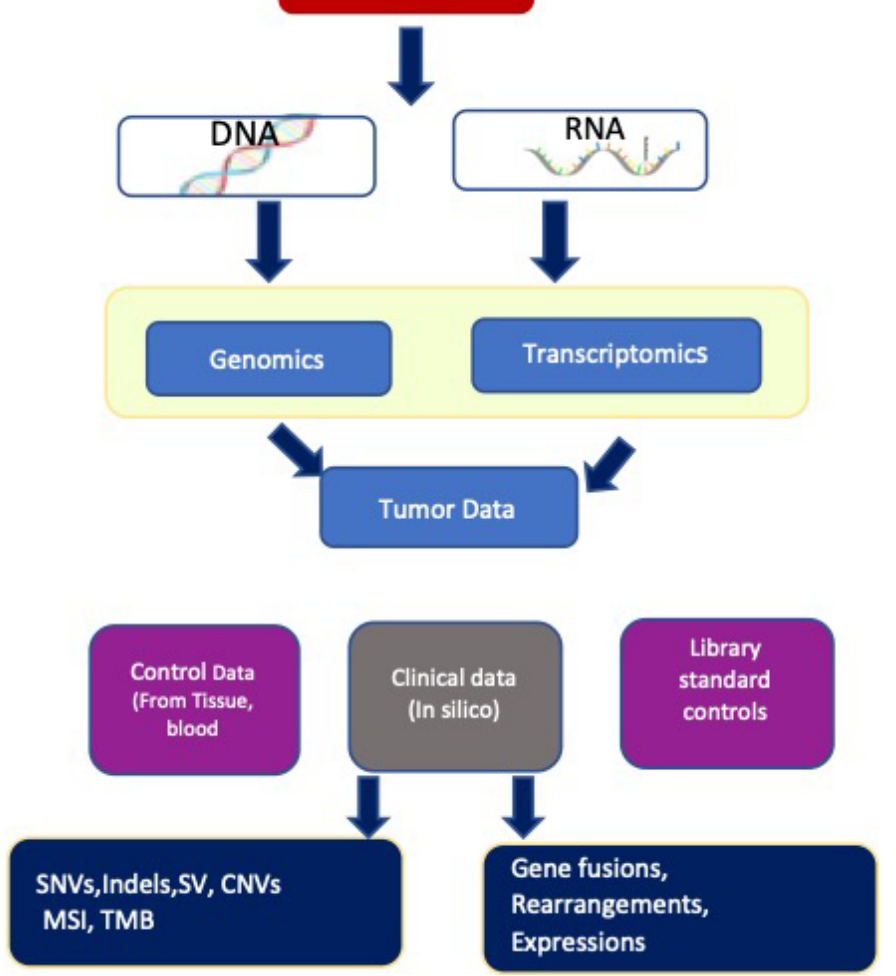

Fig. 1 The Basic NGS workflow encompassing pre- and postanalytical parameters. CNV, copy number variation; NGS, next-generation sequencing; SNV, single-nucleotide variants.

\section{Approaches for Target Enrichment}

Amplicon-based methods are convenient for limited small gene panels but are prone to unbalanced sequence coverage throughout region of interest (ROI) and artifacts, such as mismatches due to randomly binding of primers, can be introduced by polymerase errors. The coordinates of the amplified regions are fixed and invariable. Amplicon-based library preparation provides count of the number of input DNA which are unique, subjected to sequencing as a result of polymerase chain reaction (PCR) inflated coverage that are inaccurate. All PCR methods are prone to allele dropout because of the SNVs region being present at sites of primer pairing, leading to shallow read depths and/or unexpected homozygosity in NGS data.

Hybridization-based target enrichment uses complementary target-specific DNA or RNA oligonucleotide "baits" to hybridize and snare genomic DNA (fragmented due to enzymes or due to physical shearing). Hybridization capture is relatively more suitable for degenerated degraded FFPE DNA, because use of affinity-based enrichment only requires partial overlap of capture probes with region of interest, wherein amplicon PCR is unable to amplify DNA fragments where either one or both of the primer binding sites are degraded. This prevents issues of allele dropout, generally observed using amplicon-based libraries. The probes hybridizing to the region of interest are within longer fragments of DNA, in the flanking regions of the target, are amplified and sequenced. Hybridization capture-based libraries have an advantage over amplicon-based libraries, as it enables interrogation of neighboring regions that may not be easily captured with specific probes.

Hybrid capture technique is dependent on Sample Base Composition. Adenine-thymine rich sequences tend to be lost through poor annealing, while regions high in High Guanine - Cytosine (GC) content can be lost due to the formation of secondary structures. Hybrid capture library preparation is more labor intensive and time consuming as compared with amplicon-based library preparation protocols. Need of skilled, well-trained personnel is an essential prerequisite for this. ${ }^{5}$

Depending on the region of interest and applications, the choice of the correct library preparation method plays an important role in the success of the NGS assay.

\section{Importance of Next-Generation Sequencing Bioinformatics Pipeline}

NGS data analysis should be performed using robust bioinformatics pipelines. A bioinformatics pipeline consists of a large array of different types of software algorithms which process the raw data generated and provide a list of sequence variants which are annotated. ${ }^{11,12}$

Identification of true genetic alterations and variants, processing of the raw data and basic alignment, and annotations of the genetic variations identified in the assay play a mammoth role in imparting diagnostic solutions using the NGS assay.

The bioinformatics pipeline comprises base calling as the initial step, where the base sequence is allocated using the signal output. This is presided by aligning the sequence reads 
to a reference genome. Generally, for panel-based sequencing, the targeted regions of interest in the genome are specified for alignment and hence simplifying the process.

However, it is extremely important for the laboratories offering NGS-based diagnostics to get the bioinformatics pipeline also validated as per the recommended guidelines. ${ }^{12}$ The bioinformatics pipeline validation should be accurate and readily useful for the clinical use and specimen, and variant types have been designed to be detected by the test. $8,11,12$

The NGS bioinformatics data should be analyzed and interpreted only by a trained qualified health care professional after obtaining appropriate training and certification in analyzing and validation of the bioinformatics pipeline as per recommended guidelines.

\section{Variant Interpretation}

A variant is considered as a biomarker which affects clinical care. The biomarker will be of clinical utility if it can predict sensitivity, toxicity to a specific therapy, resistance or toxic response to treatment, and alteration in the gene functionality which can be addressed by use of approved or in trial drugs. The variant/biomarker acts as criterion for inclusion in clinical trials, suggesting prognosis of disease, helps in making a diagnosis of cancer, or needs implementation of continuous monitoring measures for early detection.

Somatic variants include SNVs, INDEL, fusions occurring from gene rearrangements, and CNVs. The findings of variant calling are usually depicted in one of the standardized ways, viz, variant call format (VCF), general feature format (alias gene finding format or generic feature format), and genomic VCF. However, the most common one being the VCF format. $7,8,13,14,15$

Every identified variant should be annotated with the available annotation sources like database of single nucleotide polymorphisms (dbSNP), 1,000 genomes, Exome Aggregation Consortium (ExAC), Catalogue of Somatic Mutations in Cancer (COSMIC), and ClinVar is a public archive with free access to reports on the relationships between human variations to identify mutations/pathogenic variants from benign variants and polymorphisms.

Based on the available evidence, as well as significance on the clinical decision-making, the variants have been classified into different categories as per the joint consensus and recommendations of AMP, ASCO, and CAP as mentioned below (-Fig. 2).

Reporting CNVs: It should be reported as amplification detected for gene XXX, no indications for the amplifications/ inconclusive, or additional testing required depending on the data. While reporting CNVs, it is important to include quantitative information such as coverage of the assay and assay limitations. The drawback of NGS-based CNV determination is to detect low-level copy number gains and/or high-level amplifications in specimens with low neoplastic cell percentages. Here, the quality metrics of the assay encompassing all the preanalytical, analytical, and postanalytical factors are very important. ${ }^{16}$

- Biomarker, level A: Prediction of resistance or response to U.S. FDA-approved therapies for a specific tumor or included in professional guidelines as diagnostic, therapeutic, and/or prognostic biomarkers for certain types of tumors.

\section{Tier I (Therapeutic, Diagnostic, Prognostic) Variants of strong clinical significance}
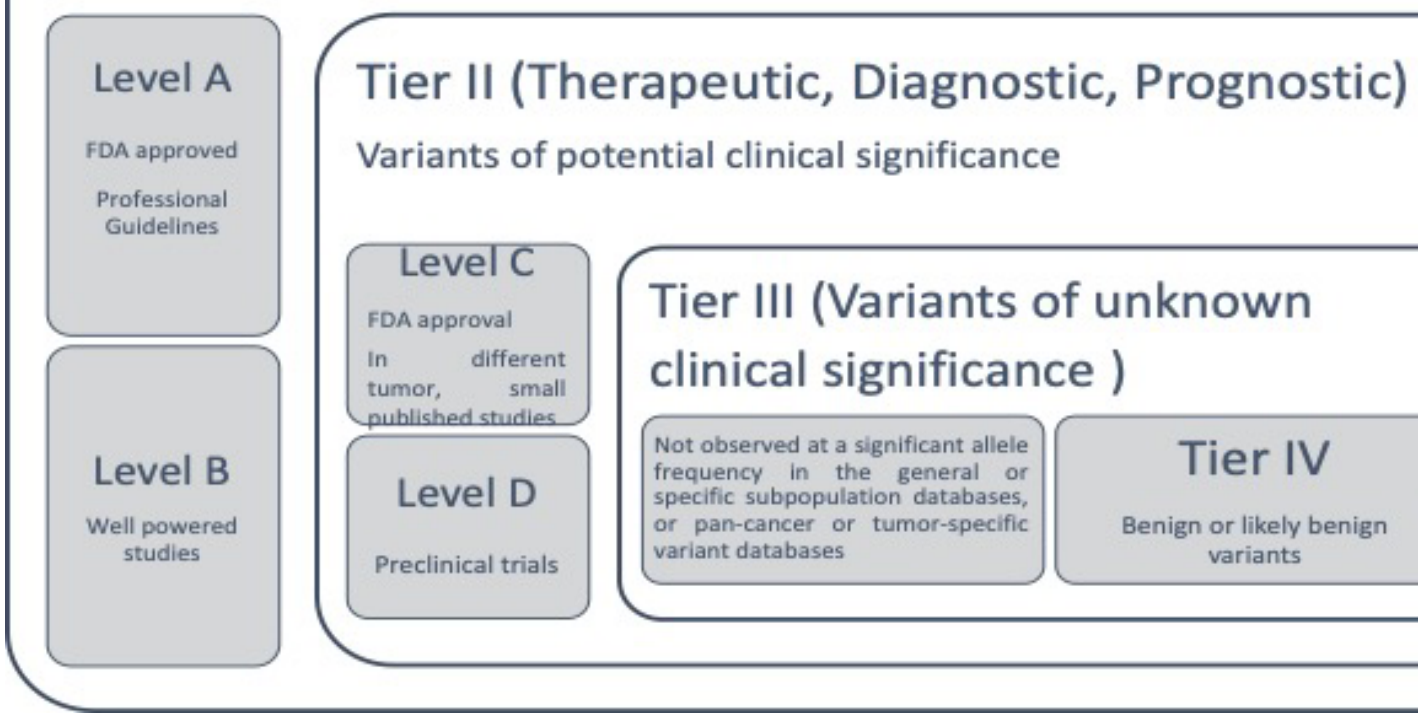

Tier III (Variants of unknown clinical significance )
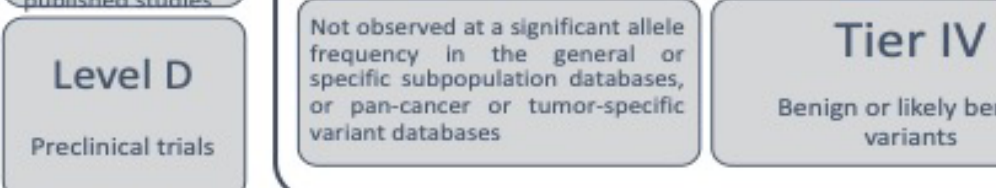

Benign or likely benign variants

Fig. 2 Tier based classification of biomarkers based on the recommendations of AMP, ACMG, and CAP.7,8 ACMG, American College of Medical Genetics and Genomics; AMP, Association for Molecular Pathology; CAP, College of American Pathologists. 
Table 3 Core quality metrics for optimum next-generation sequencing assay

\begin{tabular}{|c|c|c|}
\hline Core quality metric & Validation parameters & Ongoing quality control \\
\hline $\begin{array}{l}\text { Nucleic acid quality and } \\
\text { quantity }\end{array}$ & $\begin{array}{l}\text { Minimum criteria to ensure accurate variant detection and } \\
\text { reproducible results depending on the established sensitiv- } \\
\text { ity of the assay }\end{array}$ & $\begin{array}{l}\text { A plan for ongoing monitoring must be } \\
\text { established }\end{array}$ \\
\hline $\begin{array}{l}\text { Library qualification and } \\
\text { quantification }\end{array}$ & $\begin{array}{l}\text { The laboratory must standardize protocols for library qualifi- } \\
\text { cation and quantification }\end{array}$ & $\begin{array}{l}\text { The fragment sizes of the library -within the } \\
\text { expected molecular weight narrow range }\end{array}$ \\
\hline Depth of coverage & $\begin{array}{l}\text { Requirements vary depending on the platform used and the } \\
\text { application. Coverage must be defined to achieve adequate } \\
\text { sensitivity and specificity }\end{array}$ & $\begin{array}{l}\text { Ongoing measures should be taken to moni- } \\
\text { tor the overall coverage and region coverage } \\
\text { in each run }\end{array}$ \\
\hline Uniformity of coverage & $\begin{array}{l}\text { The required level of coverage across the targeted regions } \\
\text { must be defined }\end{array}$ & $\begin{array}{l}\text { The uniformity of coverage must be moni- } \\
\text { tored and compared with the levels estab- } \\
\text { lished during validation }\end{array}$ \\
\hline GC bias & $\begin{array}{l}\text { GC content affects sequencing efficiency and the uniformity } \\
\text { of coverage of the targeted regions. The extent of GC bias } \\
\text { should be should be determined during validation }\end{array}$ & $\begin{array}{l}\text { GC bias should be monitored with every run } \\
\text { to detect changes in test performance or } \\
\text { sample quality issues }\end{array}$ \\
\hline Base call quality scores & $\begin{array}{l}\text { The laboratory must establish acceptable raw base call qual- } \\
\text { ity score thresholds for the assay during validation }\end{array}$ & $\begin{array}{l}\text { Quality scores and quality of signal/noise ratio } \\
\text { should be monitored in every run }\end{array}$ \\
\hline Mapping quality & $\begin{array}{l}\text { Parameters for mapping quality must be established during } \\
\text { validation } \\
\text { Steps should be established to filter reads that map to } \\
\text { nontargeted regions }\end{array}$ & $\begin{array}{l}\text { The proportion of reads that do not map to } \\
\text { target regions must be monitored during } \\
\text { each run }\end{array}$ \\
\hline Duplication rate & $\begin{array}{l}\text { Acceptable parameters for maximum duplication rate } \\
\text { should be established for each assay }\end{array}$ & $\begin{array}{l}\text { The duplication rate should be monitored in } \\
\text { every run and for each sample independently }\end{array}$ \\
\hline Strand bias & $\begin{array}{l}\text { Laboratory must define the tolerance level for strand bias } \\
\text { and outline specific criteria for when alternate testing should } \\
\text { be instituted }\end{array}$ & $\begin{array}{l}\text { The degree of strand bias must be monitored } \\
\text { in all samples }\end{array}$ \\
\hline
\end{tabular}

- Biomarker, level B: predicting resistance or response to a therapy based on well-documented and well-powered studies with consensus from key opinion makers in the field, or prognostic significance, and/or have diagnostic significance of certain diseases based on well-powered studies with expert opinion and recommendations.

- Biomarker, level C: Predicting resistance or response to therapies approved by FDA or professional societies for a different tumor type (i.e., off-label drug) used as inclusion criteria in clinical trials or have diagnostic and/or prognostic significance depending on the outcome of several small studies.

- Biomarker, level D: Possible therapeutic significance based on findings of preclinical studies, or may aid in diagnosis and/or prognosis themselves or in combination with other biomarkers depending on the outcome of many small studies or multiple case reports with no expert consensus. ${ }^{7,8}$

\section{Interpretation and Reporting Recommendations}

Variant should be classified into the four-tiered system. Tiers I to III should be reported in decreasing order of clinical significance. Tier IV or "benign/likely benign" variants/alterations need not be included in the report as per recommendations; however, it should be made available. The "negatives" should be mentioned in a disease-specific manner. Identified genomic alterations should be annotated and reported as per the HUGO Gene Nomenclature (http://www.genenames.org).

Clinical significance of the variant should be clearly described in the interpretation. Evidence utilized for categorization of variants needs to be illustrated in the interpretation.
References, such as publications, and database should be appropriately cited for further information. If confirmation of a variant is performed, method of confirmation and the result should be explained. ${ }^{7,17}$

Postanalytical factors: Once the data are analyzed, the release of the NGS report is an important step. Report format needs to be static and the date of report release should be explicitly mentioned. These need not be automatically recalled and/or reissued with the change in medical knowledge. Report should clearly mention the gene panel used, pre- and post-analytical details, methodology used, and performance parameters (assay limit of detection, assay limitation, sequencing depth coverage, and annotations used).

\section{Validation of the Next-Generation Sequencing Runs}

Using this high-end technology, validation and QC play an important role. Use of appropriate controls is extremely important in every NGS run for a successful validation. The commercial reference controls with validation can be performed using different levels of variant allele frequencies. ${ }^{16} \mathrm{~A}$ reagent control excluding template must be used for library setup and should be incorporated in every run to ensure absence of carryover contamination or contamination in the reagents.

Validation of the assay will include determination of the following quality parameters:

- Positive predictive value: Positive predictive value (PPV) is the true positives which are the proportion of detected 
variants. Knowledge of all true variants is important and the actual presence of genetic variants can be estimated by using reference samples. PPV should be mentioned for each variant (e.g., SNV, small INDELs, large INDELs, CNAs, and SVs). ${ }^{6,18}$

- Limit of detection: The lower limit of detection (LLOD) is defined as minor allele fraction where $95 \%$ of samples would be detected. LLOD for every variant needs to be established. ${ }^{2,19}$

- How many samples to run for the validation: there are no specific recommendations as such for testing exact number of samples. ACMG has no specific recommendations as to how many samples need to run. CAP recommends at least 20 samples need to run and $\mathrm{CDC}$ recommends nearly 30 samples. The New York State Depart of Health (NYSDOH) recommends well-characterized cell line (e.g., NA12878) plus 50 patient samples and for "full validation," 10 positive samples of each variant type in each region must be analyzed. 2,6,18,19,20 The core quality metrics which will impact the NGS run has been summarized in - Table 3 with excerpts from AMP guidelines. ${ }^{6}$

\section{Important Points}

Some important points to consider are as follows:

- Age of the FFPE block

- Representativeness of tumor and its content

- Optimization of nucleic acid extraction protocols

- Standardization of quality and yield of the nucleic acid

- Clear understanding of the NGS workflow

- It is always important to run the tests in duplicates (technical replicates) to ensure the reproducibility of the results.

- The errors should be minimized and confidence of identifying the mutation should be high for the assay to have its true predictive value.

- To achieve 95\% reliability and 95\% confidence of the results, nearly 59 samples for a specified molecular alteration should be tested. ${ }^{6}$

\section{Role of Molecular Tumor Board}

The structured annotation and interpretation of huge NGS data, leading to translate molecular alterations into clinical indications, remains a daunting task and these issues are addressed with the introduction of the Molecular Tumor Board (MTB). ${ }^{21}$ The MTBs are multidisciplinary meetings where these molecular reports are discussed for precision management of patients from all perspectives (diagnostic radiology, histopathology, molecular pathology, surgical, medical, and radiation oncology team) to reach a consensus on understanding the molecular biomarkers, further clinical implications and consider alternative treatment options for patients who are beyond standard-of-care approaches. ${ }^{22}$

\section{Conclusion}

To conclude, molecular diagnostics is a dynamic field with continuous evolution of testing algorithms and technology. The knowledge of biomarkers needs to be constantly updated with the novel alterations or variants of potential significance being unraveled. Personalized genome medicine has reached a stage where there is no concept of one size fits all. Hence, the onus is on the laboratories performing these tests to judiciously use the clinical material and perform the test as per the recommended practice after thorough validation and analysis of the data. The paramount responsibility is borne by molecular diagnostics professional who should correctly identify and classify the variant and release the NGS report. The testing laboratories make use of the recommended guidelines, as well as relevant literature as a learning tool, to expand the testing capabilities and utilize them effectively for better patient care management.

\section{Conflict of Interest}

None declared.

\section{References}

1 Shen T, Pajaro-Van de Stadt SH, Yeat NC, Lin JC-H. Clinical applications of next generation sequencing in cancer: from panels, to exomes, to genomes. Front Genet 2015;6:215

2 Richards S, Aziz N, Bale S, et al. ACMG Laboratory Quality Assurance Committee. Standards and guidelines for the interpretation of sequence variants: a joint consensus recommendation of the American College of Medical Genetics and Genomics and the Association for Molecular Pathology. Genet Med 2015;17(5):405-424

$3 \mathrm{Xu} \mathrm{J}$, Gong B, Wu L, Thakkar S, Hong H, Tong W. Comprehensive assessments of RNA-seq by the SEQC consortium: FDAled efforts advance precision medicine. Pharmaceutics 2016;8(1):E8

4 Zhang X, Liang Z, Wang S, et al. Application of next-generation sequencing technology to precision medicine in cancer: joint consensus of the Tumor Biomarker Committee of the Chinese Society of Clinical Oncology. Cancer Biol Med 2019;16(1):189-204

5 Cheng DT, Mitchell TN, Zehir A, et al. Memorial sloan kettering- integrated mutation profiling of actionable cancer targets (MSK-IMPACT): A hybridization capture-based next-generation sequencing clinical assay for solid tumor molecular oncology. J Mol Diagn 2015;17(3):251-264

6 Jennings LJ, Arcila ME, Corless C, et al. Guidelines for validation of next-generation sequencing-based oncology panels: a joint consensus recommendation of the Association for Molecular Pathology and College of American Pathologists. J Mol Diagn 2017;19(3):341-365

7 Richards S, Aziz N, Bale S, et al. Standards and guidelines for the interpretation of sequence variants: a joint consensus recommendation of the American College of Medical Genetics and Genomics and the Association for Molecular Pathology. Genet Med 2015;17(5):405-424

8 Li MM, Datto M, Duncavage EJ, et al. Standards and guidelines for the interpretation and reporting of sequence variants in cancer: a joint consensus recommendation of the Association for Molecular Pathology, American Society of Clinical Oncology, and College of American Pathologists. J Mol Diagn 2017;19(1):4-23 
9 Aziz N, Zhao Q Bry L, et al. College of American Pathologists' laboratory standards for next-generation sequencing clinical tests. Arch Pathol Lab Med 2015;139(4):481-493

10 El-Deiry WS, Goldberg RM, Lenz HJ, et al. The current state of molecular testing in the treatment of patients with solid tumors, 2019. CA Cancer J Clin 2019;69(4):305-343

$11 \mathrm{Li} \mathrm{H}$, Durbin R. Fast and accurate long-read alignment with Burrows-Wheeler transform. Bioinformatics 2010;26(5):589-595

12 Roy S, Coldren C, Karunamurthy A, et al. Standards and guidelines for validating next-generation sequencing bioinformatics pipelines: a joint recommendation of the Association for Molecular Pathology and the College of American Pathologists. J Mol Diagn 2018;20(1):4-27

13 Larson DE, Harris CC, Chen K, et al. SomaticSniper: identification of somatic point mutations in whole genome sequencing data. Bioinformatics 2012;28(3):311-317

14 Garrison E, Marth G. Haplotype-based variant detection from short-read sequencing. Available at: https://arxiv.org/ pdf/1207.3907.pdf. Accessed June 29, 2021

15 Schrijver I, Farkas DH, Gibson JS, Lyon E; AMP Executive Committee. The evolving role of the laboratory professional in the age of genome sequencing: a vision of the association for molecular pathology. J Mol Diagn 2015;17(4):335-338

16 Eijkelenboom A, Tops BB, van den Berg A, et al. Recommendations for the clinical interpretation and reporting of copy number gains using gene panel NGS analysis in routine diagnostics. Virchows Arch 2019;474(6):673-680

17 Gulley LM, Braziel RM, Halling KC, et al. Molecular Pathology Resource Committee, College of American Pathologists. Clinical laboratory reports in molecular pathology. Arch Pathol Lab Med 2007;131(6):852-863

18 Meldrum C, Doyle MA, Tothill RW. Next-generation sequencing for cancer diagnostics: a practical perspective. Clin Biochem Rev 2011;32(4):177-195

19 Luthra R, Chen H, Roy-Chowdhuri S, Singh RR, Next-generation sequencing in clinical molecular diagnostics of cancer: advantages and challenges 2015;32(4):177-195

20 Feldman GL, Schrijver I, Lyon E, Palomaki GE; CAP/ACMG Biochemical and Molecular Genetics Resource Committee. Results of the College of American Pathology/American College of Medical Genetics and Genomics external proficiency testing from 2006 to 2013 for three conditions prevalent in the Ashkenazi Jewish population. Genet Med 2014;16(9):695-702

21 Perera-Bel J, Hutter B, Heining C, et al From somatic variants towards precision oncology: Evidence-driven reporting of treatment options in molecular tumor boards. Genome Med 2018;10(1):18

22 Luchini C, Lawlor RT, Milella M, Scarpa A. Molecular tumor boards in clinical practice. Trends Cancer 2020;6(9):738-744 